\title{
Estenose da fossa intercondilar após estabilização articular com retalho de fáscia lata em cães
}

\author{
Intercondylar fossa stenosis after joint stabilization using a fascial strip in dogs
}

\author{
André Luis Selmi ${ }^{\mathrm{I}, \mathrm{I} *}$ João Guilherme Padilha Filho"II Bruno Testoni Lins ${ }^{\mathrm{II}}$ \\ Andrigo Barboza De Nardi ${ }^{\mathrm{I}}$ Bianca Mota Penteado ${ }^{\mathrm{I}}$
}

\section{RESUMO}

\begin{abstract}
Determinaram-se os índices de largura da fossa intercondilar (FI), após transecção do ligamento cruzado cranial em nove cães adultos submetidos à estabilização articular com retalho de fáscia lata. Os joelhos foram alocados em dois grupos, sendo o joelho direito (GI) submetido à incisuroplastia troclear (ITR) e posterior estabilização articular, $e$ o joelho esquerdo submetido somente à substituição ligamentar $(G C)$. Cada grupo foi dividido em três subgrupos correspondentes aos momentos de eutanásia aos 30, 90 e 180 dias de pós-operatório. Os índices de largura da FI foram determinados, macroscópica e radiograficamente, pela mensuração da abertura cranial da FI nos terços cranial, médio e caudal, e indexados em relação à largura epicondilar. Observou-se aumento significativo dos índices macroscópicos e radiográficos nas articulações do GI, sendo estes estatisticamente diferentes daqueles das articulações de GC. Não foi observada estenose intercondilar nos joelhos de GC após a estabilização articular. Conclui-se que a estabilização articular com retalho de fáscia lata preveniu a estenose da fossa intercondilar, e que a ITR promoveu o alargamento permanente dessa estrutura.
\end{abstract}

Palavras-chave: estenose intercondilar, fossa intercondilar, ligamento cruzado cranial, cães.

\section{ABSTRACT}

Intercondylar fossa width indexes (IFWI) were determined in nine adult dogs submitted to intercondylar notchplasty (IN) after transection of the cranial cruciate ligament (CCL) followed by a fascial strip stabilization. The right stifle was submitted to IN followed by fascial strip reconstruction of the CCL (GI) while in the left stifle IN was not performed $(G C)$. Each group was then divided into three subgroups which corresponded to time of euthanasia at 30, 90 and 180 days after surgery. IFWI were determined, both macroscopically and radiographically, by measuring the cranial outlet of the intercondylar fossa in relation to the epicondylar width. A significant increase was observed in indexes of GI following IN, and these differed from indexes of GC throughout the evaluation period. It was concluded that articular repair using a fascia strip prevented stenosis of the intercondylar fossa, and that IN caused a permanent widening of it.

Key words: intercondylar notch stenosis, intercondylar fossa, cranial cruciate ligament, dogs.

\section{INTRODUÇÃO}

A importância da FI na ruptura do ligamento cruzado cranial (RLCCr) foi identificada por volta de 1980 em humanos e na década de 90 em cães (SOURYAL et al. 1988; FITCH et al., 1995a; MONTGOMERY et al., 1995). Postula-se que a formação de osteófitos sobre a margem medial do côndilo lateral do fêmur, resultante da doença articular degenerativa secundária a RLCCr, cause estenose intercondilar, favorecendo a abrasão do ligamento e rompimento precoce dessa estrutura (AIKEN et al., 1995; FITCH et al., 1995a; FITCH et al., 1995b; MONTGOMERY et al., 1995; LAPRADE et al., 1998; MANN et al., 1999; SELMI et al., 2007; LEWIS et al., 2008).

${ }^{\mathrm{I}}$ Mestrado em Medicina Veterinária de Pequenos Animais, Universidade de Franca (UNIFRAN), 14404-600, Franca, SP, Brasil. Email: andre_selmi@yahoo.com.br.*Autor para correspondência.

"Curso de Medicina Veterinária, Universidade Anhembi Morumbi (UAM), São Paulo, SP, Brasil.

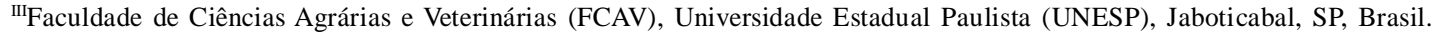


A estenose intercondilar causada pela formação de osteófitos foi observada, experimentalmente, por FITCH et al. (1995b) após incisuroplastia troclear (ITR), acompanhada ou não da transecção do ligamento cruzado cranial em cães, e por LA PRADE et al. (1998) após realização de ITR em articulações estáveis, sugerindo que o estreitamento da FI, a longo prazo, especialmente em articulações instáveis, decorre da falta de contato da porção lateral da FI com estruturas intra-articulares.

AIKEN et al. (1995) observaram que os índices de largura intercondilar eram significativamente menores em cães com RLCCr, quando comparados àqueles obtidos a partir de articulações estáveis, e sugeriram que reconstruções intra-articulares sejam precedidas de ITR, que consiste na remoção cirúrgica da porção medial do côndilo lateral do fêmur que pode entrar em contato com o LCCr, ou seu substituto, predispondo-o à ruptura (MANN et al., 1999). SELMI et al. (2007) compararam os efeitos da ITR sobre a estabilização intra-articular com fáscia lata em cães e notaram similitude entre as alterações clínicas e radiográficas em avaliações pós-operatórias. Mais recentemente, LEWIS et al. (2008) empregaram tomografia computadorizada para avaliação da fossa intercondilar em cães clinicamente normais e com RLCCr e descreveram resultados similares àqueles obtidos por imagens radiográficas.

A despeito do pouco interesse na estenose intercondilar subsequente à RLCCr em cães, essa alteração tem sido implicada no insucesso pósoperatório após a estabilização intra-articular em cães, em especial, após a utilização de substitutos sintéticos do LCCr (VASSEUR et al., 1996; SELMI et al., 2002; ACKER, 2009).

Objetivou-se com este estudo determinar, macroscópica e radiograficamente, os índices de largura intercondilar no joelho de cães submetidos à estabilização intra-articular com retalho de fáscia lata, precedida ou não de ITR.

\section{MATERIAL E MÉTODOS}

Foram utilizados nove cães hígidos, sem raça definida, machos ou fêmeas, adultos, com massa corporal média: $20,9 \pm 4,8 \mathrm{~kg}$, provenientes do Centro de Controle de Zoonoses. Os animais foram colocados em canis individuais com aproximadamente $1,5 \mathrm{~m}^{2} \mathrm{de}$ área. Receberam ração comercial e água ad libitum. Para o desenvolvimento deste estudo, os cães tiveram o joelho direito submetido à desmotomia do cruzado cranial, ITR e posterior estabilização articular com retalho de fáscia lata pela técnica under-and-over, proposta por COETZEE (1993) (GI), enquanto que o joelho esquerdo foi submetido à desmotomia do $\mathrm{LCCr}$ e à substituição ligamentar (GC). Os cães foram agrupados em três subgrupos correspondentes ao período de eutanásia no $30^{\circ}, 90^{\circ}$ e $180^{\circ}$ dia pósoperatório.

Para realização dos procedimentos cirúrgicos, os animais foram mantidos em jejum hídrico e alimentar por 12 horas. A medicação pré-anestésica com maleato de acepromazina ${ }^{\mathrm{a}}\left(0,1 \mathrm{mg} \mathrm{kg}^{-1}-\mathrm{IV}\right)$ foi seguida pela indução anestésica com propofol ${ }^{\mathrm{b}}(6 \mathrm{mg}$ $\mathrm{kg}^{-1}$ - IV) e então os animais foram intubados e a anestesia mantida em circuito semifechado, empregando-se sevoflurano ${ }^{c}$ vaporizado em oxigênio a $100 \%$. Todos cães foram submetidos à analgesia epidural com cloridrato de morfina ${ }^{\mathrm{d}}\left(0,1 \mathrm{mg} \mathrm{kg}^{-1}\right)$, cloridrato de lidocaína ${ }^{\mathrm{e}}\left(1 \mathrm{mg} \mathrm{kg}^{-1}\right)$ e bupivacaína ${ }^{\mathrm{f}}$ $\left(0,51 \mathrm{mg} \mathrm{kg}^{-1}\right)$.

Abordou-se a articulação por incisão parapatelar lateral, seguida da remoção do LCCr. No GI, realizou-se a ITR, que consistiu no desgaste de aproximadamente $4 \mathrm{~mm}$ do aspecto medial do côndilo lateral do fêmur, com auxílio de fresa óssea. Posteriormente, o joelho foi estabilizado com retalho de fáscia lata, conforme técnica descrita por COETZEE (1993), consistindo em um retalho intra-articular e extraarticular. Após estabilização articular, os tecidos periarticulares foram aproximados, respeitando-se os planos anatômicos. No GC, o procedimento cirúrgico foi análogo, exceto pela ausência da incisuroplastia troclear. As cirurgias foram realizadas em momentos subsequentes.

Um adesivo de liberação gradativa de fentanilg foi aplicado à região lombar, imediatamente ao término da cirurgia. A administração de cetoprofeno ${ }^{\mathrm{h}}$ foi mantida durante quatro dias subsequentes ao ato cirúrgico. Diariamente, efetuou-se limpeza da ferida cirúrgica até a retirada dos pontos no décimo dia pósoperatório.

Para determinação da largura intercondilar e posterior cálculo dos índices radiográficos e macroscópicos, foi empregada a técnica descrita por FITCH et al. (1995a). Os joelhos foram radiografados em incidência caudocranial, com a tíbia em flexão aproximada de $20^{\circ}$ e oblíquas a $7^{\circ}$ em relação ao fêmur. A mensuração da largura da FI foi realizada nos terços cranial, médio e caudal (Figura 1). O procedimento foi realizado imediatamente antes (T-1) e após a cirurgia (T1), e aos 30 (T30), 90 (T90) e 180 dias (T180) subsequentes. A determinação macroscópica dos índices foi realizada durante o ato cirúrgico, imediatamente antes da transecção do LCCr, sendo esses valores considerados equivalentes aos valores 


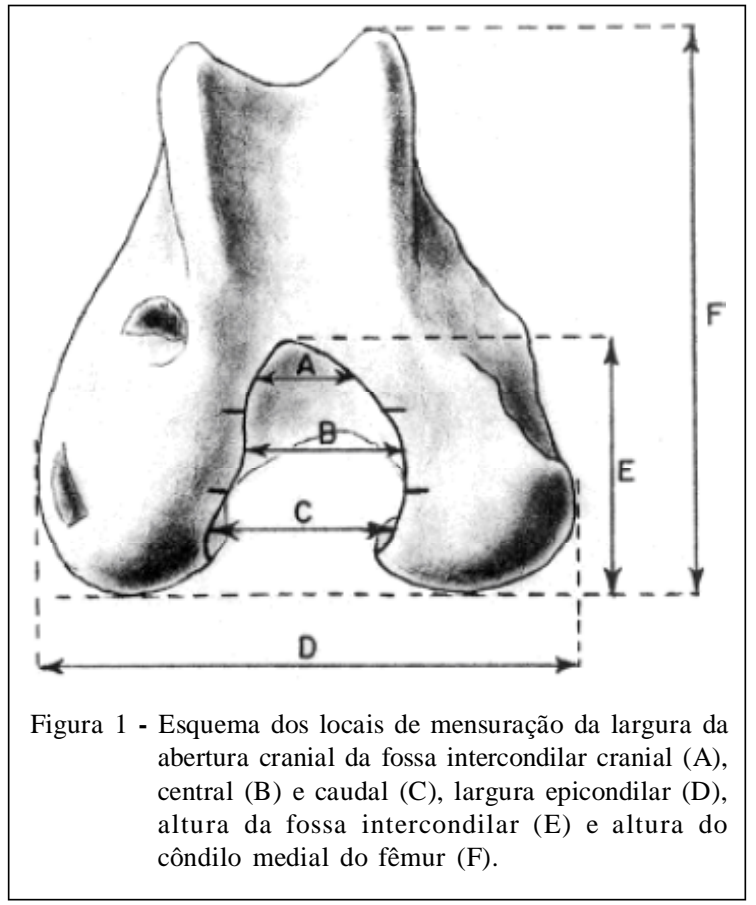

pré-cirúrgicos radiográficos para fins de comparação. As demais mensurações macroscópicas e radiográficas foram obtidas nos respectivos momentos de eutanásia para cada subgrupo. Todas as exposições radiográficas foram realizadas com os animais sob efeito de anestesia geral, empregando-se acepromazina ( $0,1 \mathrm{mg} \mathrm{kg}^{-1}$ - IV), seguida de tiopental sódico $\left(12,5 \mathrm{mg} \mathrm{kg}^{-1}\right.$ - IV). Nos momentos de avaliação, determinou-se também o índice macroscópico de altura da fossa intercondilar (IMAFI), considerando como altura da FI a distância entre o ponto mais cranial da abertura cranial da FI e a tangente da superfície caudal do côndilo medial. Para cálculo do IMAFI, dividiu-se o valor da altura da fossa intercondilar pela altura do côndilo medial, medida de seu aspecto mais caudal até sua eminência mais proximal (FITCH et al., 1995a). Todas as mensurações foram realizadas utilizando-se de paquímetro'. Avaliouse também o formato da abertura cranial da FI, sendo estas classificadas em "crista de onda", obcônicas, quadradas ou semicirculares (FITCH et al., 1995a; MONTGOMERY et al., 1995), e a integridade do neoligamento durante os momentos de eutanásia, correspondentes a T30, T90 e T180.

Os índices macroscópicos de largura da fossa intercondilar obtidos após ITR foram considerados análogos àqueles obtidos radiograficamente no pós-operatório imediato (T1). As médias foram analisadas por ANOVA para medidas repetidas seguida de teste de Tukey, para comparação dentro dos grupos e entre cada grupo para variáveis idênticas. Para comparação das médias macroscópicas e radiográficas obtidas dentro de cada grupo, utilizouse $o$ teste de t pareado. Consideraram-se significativos valores de $\mathrm{P}<0,05$.

\section{RESULTADOS E DISCUSSÃO}

Previamente à cirurgia, as aberturas craniais das fossas intercondilares apresentavam-se com formato quadrado (oito joelhos) ou cônico (11 joelhos). Nos animais submetidos à ITR, a abertura cranial da FI adquiriu formato semicircular. Nos respectivos momentos de eutanásia, em todas as articulações, observou-se alteração do formato da abertura cranial da FI, sendo que, naqueles animais nos quais foi realizada a ITR, o formato final apresentou-se cônico (dois joelhos) ou semicircular (sete joelhos), enquanto que, nas articulações do grupo controle, o formato apresentou-se em "crista de onda" (nove joelhos), evidenciando a formação de osteófitos, principalmente no aspecto medial do côndilo lateral do fêmur. Referente ao neoligamento, observou-se integridade do mesmo em todas as articulações de GI e GC, sem que fossem notados quaisquer sinais de abrasão, fragmentação ou ruptura.

As alterações morfológicas observadas na abertura cranial da FI dos joelhos de GI, em todos os momentos de avaliação, confirmaram as citações de FITCH et al. (1995b), que relataram a manutenção da forma dessa região após realização de ITR em cães com joelhos estáveis. Esse achado possivelmente ocorreu devido à estabilização propiciada pela disposição intra e extra-articular do retalho de fáscia lata, apesar de que, no GC, a abertura cranial apresentou discreto estreitamento, levando à alteração morfológica devido à formação de osteófitos no aspecto medial do côndilo lateral do fêmur, conforme observações relatadas por FITCH et al. (1995b), MONTGOMERY et al. (1995) e FITCH et al. (1996), possivelmente decorrentes do insulto articular durante a cirurgia ou secundariamente à instabilidade residual, ou ainda devido à falta de contato do neoligamento com a porção lateral da FI(LA PRADE et al.,1998).

Previamente às cirurgias, não foram observadas diferenças significativas entre os índices macroscópicos (Tabela 1) ou radiográficos (Tabela 2) nas articulações de GC ou GI, tampouco diferenças entre os índices macroscópicos e radiográficos dentro de cada grupo, evidenciando a homogeneidade da amostra e a correlação entre as mensurações macroscópicas e radiográficas.

Observou-se aumento significativo dos índices macroscópicos e radiográficos, imediatamente 
Tabela 1 - Médias e desvios-padrão dos índices macroscópicos da largura intercondilar cranial (IMLICr), central (IMLICe) e caudal (IMLICa) dos membros pélvicos submetidos à desmotomia do LCCr e substituição ligamentar com retalho de fáscia lata associada à incisuroplastia troclear (GI) ou não (GC), no período pré-operatório (T-1), pós-operatório imediato (T1) e aos 30, 90 e 180 dias pós-operatórios (T30, T90 e T180, respectivamente).

\begin{tabular}{|c|c|c|c|c|c|c|}
\hline \multicolumn{2}{|c|}{ Variáveis macroscópicas } & \multirow[b]{2}{*}{$\mathrm{T}-1$} & \multirow[b]{2}{*}{$\mathrm{T} 1$} & \multirow[b]{2}{*}{ T30 } & \multirow[b]{2}{*}{ T90 } & \multirow[b]{2}{*}{$\mathrm{T} 180$} \\
\hline & & & & & & \\
\hline \multirow{3}{*}{ GI } & IMLICr & $0,196 \pm 0,03$ & $0,274 \pm 0,02^{* \mathrm{a}}$ & $0,266 \pm 0,04^{* a}$ & $0,265 \pm 0,04^{* a}$ & $0,262 \pm 0,03^{* a}$ \\
\hline & IMLICe & $0,227 \pm 0,04$ & $0,347 \pm 0,04^{* b}$ & $0,344 \pm 0,02^{* b}$ & $0,331 \pm 0,05^{* b}$ & $0,330 \pm 0,03^{* b}$ \\
\hline & IMLICa & $0,311 \pm 0,05$ & $0,356 \pm 0,04^{* \mathrm{c}}$ & $0,354 \pm 0,02^{*_{\mathrm{c}}}$ & $0,339 \pm 0,05^{*_{\mathrm{c}}}$ & $0,330 \pm 0,04^{* \mathrm{c}}$ \\
\hline \multirow{3}{*}{ GC } & IMLICr & $0,192 \pm 0,04$ & $0,192 \pm 0,04^{\mathrm{a}}$ & $0,191 \pm 0,02^{\mathrm{a}}$ & $0,191 \pm 0,04^{\mathrm{a}}$ & $0,191 \pm 0,02^{\mathrm{a}}$ \\
\hline & IMLICe & $0,239 \pm 0,05$ & $0,244 \pm 0,06^{\mathrm{b}}$ & $0,248 \pm 0,02^{\mathrm{b}}$ & $0,249 \pm 0,08^{b}$ & $0,244 \pm 0,04^{\mathrm{b}}$ \\
\hline & IMLICa & $0,310 \pm 0,07$ & $0,310 \pm 0,07^{\mathrm{c}}$ & $0,309 \pm 0,04^{\mathrm{c}}$ & $0,304 \pm 0,09^{c}$ & $0,299 \pm 0,05^{\mathrm{c}}$ \\
\hline
\end{tabular}

Médias seguidas por * são significativamente diferentes de $\mathrm{T}-1(\mathrm{P}<0,01)$. Médias seguidas pela mesma letra são significativamente diferentes entre os grupos, dentro de cada período de avaliação $(\mathrm{P}<0,05)$.

após a realização da ITR, com diminuição progressiva dos valores ao longo do estudo em GI, enquanto que, em GC, os valores médios dos índices macroscópicos e radiográficos diferiram significativamente daqueles obtidos dos joelhos de GI, entretanto, não entre os momentos de avaliação. Apesar dessa diferença, não foi observada, ao longo do estudo, em ambos os grupos, diminuição dos índices que pudessem sugerir estenose da FI; contudo, FITCH et al. (1995b) e FITCH et al. (1996) descreveram estenose intercondilar em joelhos estáveis submetidos a ITR sem no entanto terem observado diferenças significativas nos índices macroscópicos ou radiográficos, enquanto que em joelhos instáveis submetidos à ITR, a estenose intercondilar foi acompanhada por redução de ambos os índices, confirmando a eficácia da estabilização cirúrgica em ambos os grupos deste estudo na prevenção da estenose da FI.

Apesar da forma da FI apresentar alteração nos momentos de observação, a ausência de estenose progressiva da estrutura, de acordo com as mensurações realizadas neste estudo, sugere que a técnica proposta por COETZEE (1993) foi adequada em manter a estabilidade articular, visto que a instabilidade é um dos fatores relacionados à formação de osteófitos na FI (FITCH et al., 1995b; FITCH et al., 1996; LA PRADE et al., 1998). De acordo com COETZEE (1993), a disposição over-the-top da fáscia lata, nas estabilizações intra-articulares, propicia posicionamento isométrico do substituto ligamentar, diminuindo as cargas cíclicas aplicadas ao neoligamento.

Tabela 2 - Médias e desvios-padrão dos índices radiográficos de largura intercondilar cranial (IRLICr), central (IRLICe) e caudal (IRLICa) dos membros pélvicos, submetidos à desmotomia do LCCr e substituição ligamentar com retalho de fáscia lata associada à incisuroplastia troclear (GI) ou não (GC), no período pré-operatório (T-1), pós-operatório imediato (T1) e aos 30, 90 e 180 dias pós-operatórios (T30, T90 e T180, respectivamente).

\begin{tabular}{|c|c|c|c|c|c|c|}
\hline \multicolumn{2}{|c|}{ Variáveis radiográficas } & \multirow[b]{2}{*}{$\mathrm{T}-1$} & \multirow[b]{2}{*}{$\mathrm{T} 1$} & \multirow[b]{2}{*}{$\mathrm{T} 30$} & \multirow[b]{2}{*}{ T90 } & \multirow[b]{2}{*}{$\mathrm{T} 180$} \\
\hline & & & & & & \\
\hline \multirow{3}{*}{ GI } & IRLICr & $0,197 \pm 0,03$ & $0,267 \pm 0,03^{* a}$ & $0,264 \pm 0,04^{*_{a}}$ & $0,263 \pm 0,03^{*_{a}}$ & $0,259 \pm 0,03^{* a}$ \\
\hline & IRLICe & $0,230 \pm 0,04$ & $0,337 \pm 0,04^{* \mathrm{~b}}$ & $0,329 \pm 0,02^{* \mathrm{~b}}$ & $0,329 \pm 0,05^{* b}$ & $0,327 \pm 0,03^{* b}$ \\
\hline & IRLICa & $0,312 \pm 0,05$ & $0,348 \pm 0,04^{* \mathrm{c}}$ & $0,345 \pm 0,02^{* \mathrm{c}}$ & $0,343 \pm 0,05^{*^{c}}$ & $0,332 \pm 0,04^{* \mathrm{c}}$ \\
\hline \multirow{3}{*}{ GC } & IRLICr & $0,198 \pm 0,04$ & $0,199 \pm 0,04^{\mathrm{a}}$ & $0,196 \pm 0,02^{\mathrm{a}}$ & $0,194 \pm 0,04^{\mathrm{a}}$ & $0,188 \pm 0,02^{\mathrm{a}}$ \\
\hline & IRLICe & $0,246 \pm 0,05$ & $0,244 \pm 0,06^{\mathrm{b}}$ & $0,232 \pm 0,02^{\mathrm{b}}$ & $0,226 \pm 0,08^{\mathrm{b}}$ & $0,221 \pm 0,04^{\mathrm{b}}$ \\
\hline & IRLICa & $0,311 \pm 0,07$ & $0,308 \pm 0,07^{\mathrm{c}}$ & $0,306 \pm 0,04^{\mathrm{c}}$ & $0,303 \pm 0,09^{c}$ & $0,301 \pm 0,05^{\mathrm{c}}$ \\
\hline
\end{tabular}

Médias seguidas por * são significativamente diferentes de T-1 ( $<<0,01)$. Médias seguidas pela mesma letra são significativamente diferentes entre os grupos, dentro de cada período de avaliação $(\mathrm{P}<0,05)$.

Ciência Rural, v.42, n.1, jan, 2012. 
Não foram observadas diferenças significativas nos índices macroscópicos entre os valores imediatamente após a ITR e nos períodos posteriores de avaliação em GC e em GI (Tabela 1). Esse mesmo comportamento foi observado nas médias dos índices radiográficos em GC e GI (Tabela 2).

Para evitar comparações infundadas entre as dimensões macroscópicas e radiográficas da FI, SOURYAL \& FREEMAN (1993) sugeriram a indexação dos valores de largura intercondilar, permitindo comparações entre pacientes de diferentes tamanhos. De fato, os valores obtidos nos momentos préoperatórios neste estudo se assemelham àqueles descritos por FITCHet al. (1995a) e FITCHet al. (1996). A semelhança entre os valores obtidos, macroscópica e radiograficamente, também foi descrita no estudo de FITCH et al. (1995a), MONTGOMERY et al. (1995) e FITCH et al. (1996) que utilizaram a indexação nas determinações da largura da fossa intercondilar em cães normais e relataram similaridade entre os valores. A vantagem do método é que ele racionaliza as mensurações obtidas, tendo aplicação macroscópica e radiográfica, e nega as diferenças de tamanho entre indivíduos (SOURYAL \& FREEMAN, 1993) e as possíveis magnificações decorrentes da técnica radiográfica (FITCH et al., 1995a; MONTGOMERY et al., 1995).

FITCH et al. (1996) compararam as mensurações obtidas na avaliação radiográfica da FI com aquelas obtidas por tomografia computadorizada e macroscopia em joelhos estáveis e instáveis. Eles descreveram que somente a abertura intercondilar cranial foi subestimada durante avaliação radiográfica ou tomográfica, em comparação com a avaliação macroscópica. No entanto, essa discrepância entre mensurações não foi observada neste estudo, uma vez que os valores macroscópicos e radiográficos, em ambos os grupos, não apresentaram diferenças estatísticas.

Recentemente, as mensurações tomográficas da FI em cães normais e com RLCCr foram descritas por LEWIS et al. (2008) e, apesar da pequena variância entre os valores obtidos naquele estudo e neste, comparações diretas entre os estudos são difíceis de serem realizadas pela diferença entre os métodos utilizados, uma vez que, neste estudo, a FI foi dividida em sentido craniocaudal e, no estudo de LEWIS et al. (2008), a FI foi dividida em sentido proximodistal.

Neste estudo, o posicionamento cranial do retalho de fáscia lata durante seu trajeto por sob o ligamento intermeniscal favoreceu o maior contato deste com o teto da FI, de forma que o desgaste da porção cranial da FI em GI, incluindo parte do teto, diminuiu o atrito entre a superfície óssea e o neoligamento. Entretanto, não foram observadas diferenças significativas entre os valores, macroscópicos ou radiográficos, do índice de altura da fossa intercondilar (Tabela 3). Provavelmente, a ausência de complicações relativas à abrasão do retalho de fáscia lata com o teto da FI em GC foi decorrente da estabilização imediata da articulação e contato do neoligamento com superfície articular íntegra, enquanto que em GI, a ITR favoreceu a falta de contato entre o neoligamento e o teto intercondilar. Tentativas de se posicionar o neoligamento através de túneis ósseos favoreceriam o deslocamento caudal sugerido por HOWELL \& TAYLOR (1993), mas predisporiam ao atrito com as bordas ósseas do túnel tibial, com consequente insucesso pós-operatório, conforme descreveram VASSEUR et al. (1996) e SELMI et al. (2002). Corroboram esses achados aqueles descritos por SELMI et al. (2007) que relataram a manutenção da amplitude de movimento em joelhos submetidos à estabilização intra-articular, precedida ou não pela ITR.

\section{CONCLUSÃO}

Baseado nos resultados obtidos, concluise que a estabilização intra-articular com retalho de fáscia lata pode prevenir a estenose da fossa intercondilar e que a incisuroplastia troclear mantém o alargamento da fossa intercondilar, durante o período de avaliação estudado.

Tabela 3 - Médias e desvios-padrão do índice macroscópico de altura da fossa intercondilar nos membros pélvicos submetidos à desmotomia do LCCr e substituição ligamentar com retalho de fáscia lata associada à incisuroplastia troclear (GI) ou não (GC), no período préoperatório (T-1), pós-operatório imediato (T1) e aos 30, 90 e 180 dias pós-operatórios (T30, T90 e T180, respectivamente).

\begin{tabular}{|c|c|c|c|c|c|}
\hline \multirow{2}{*}{ Altura da fossa intercondilar } & & & aran & & \\
\hline & $\mathrm{T}-1$ & $\mathrm{~T} 1$ & $\mathrm{~T} 30$ & T90 & $\mathrm{T} 180$ \\
\hline GI & $0,43 \pm 0,06$ & $0,46 \pm 0,04$ & $0,45 \pm 0,03$ & $0,45 \pm 0,07$ & $0,44 \pm 0,06$ \\
\hline GC & $0,43 \pm 0,03$ & $0,43 \pm 0,03$ & $0,43 \pm 0,07$ & $0,43 \pm 0,08$ & $0,43 \pm 0,05$ \\
\hline
\end{tabular}




\section{FONTES DE AQUISIÇÃO}

a - Acepran. Univet S.A. SP.

b - Propoabbott. Abbott do Brasil. SP.

c - Sevorane. Abbott do Brasil. SP

d - Dimorf. Cristália. SP.

e - Xilestesin. Cristália. SP.

f - Bupivacaína. Cristália. SP.

g - Durogesic. Jansen \& Cillag. Canadá.

h - Ketofen. Merial. SP.

i - Thionembutal. Abbott do Brasil. SP

j - Mitutoyo. Japão.

\section{COMITÊ DE ÉTICA E BIOSEGURANÇA}

Protocolo n.124/00.

\section{REFERÊNCIAS}

ACKER, R. Prosthetic cruciate repair. In: KYON SYMPOSIUM, 2009, Zurique, Suiça. Proceedings.... Disponível em: <http://www.kyon.ch/wp-content/uploads/2009/ $10 /$ S Y MP_ 2008 _PROSTHESIS_ 16 - Acker-CrC1Prosthesis.pdf $>$. Acesso em: 23 maio, 2010.

AIKEN, S.W. et al. Intercondylar notch width in dogs with and without cranial cruciate ligament injuries. Veterinary and Comparative Orthopedics and Traumatology, v. 8, p.128 132, 1995. Disponível em: <http://www.scielo.br/ scielo.php? script=sci_art text \& pid=S 0103 84782007000500022>. Acesso em: 23 maio, 2010. doi: $10.1590 / \mathrm{S} 0103-84782007000500022$

COETZEE, G.L. An in vitro comparison of two replacement techniques utilizing fascia lata after cranial cruciate ligament transection in the dog. Veterinary and Comparative Orthopedics and Traumatology, v.6, p.85-93, 1993.

FITCH, R.B. et al. The intercondylar fossa of the normal canine stifle: an anatomic and radiographic study. Veterinary Surgery, v.24, p.148-155, 1995a.

FITCH, R.B. et al. The effect of intercondylar notchplasty on the normal canine stifle. Veterinary Surgery, v.24, p.156164, 1995b.

FITCH, R.B. et al. Radiographic and computed tomographic evaluation of the canine intercondylar fossa in normal stifles and after notchplasty in stable and unstable stifles. Veterinary Radiology and Ultrasound, v.4, p.266-274, 1996. Disponível em: <http://onlinelibrary.wiley.com/doi/10.1111/j.17408261.1996.tb01229.x/abstract>. Acesso em: 23 maio, 2010. doi: $10.1111 / \mathrm{j} .1740-8261.1996 . t b 01229 . x$.

HOWELL, S.M.; TAYLOR, M.A. Failure of reconstruction of the anterior cruciate ligament due to impingement by the intercondylar roof. Journal of Bone and Joint Surgery, v.75-A, n.7, p.1044-1055, 1993.

LAPRADE, R.F. et al. The effects of aggressive notchplasty on the normal knee in dogs. American Journal of Sports Medicine, v.26, n.2, p.193-200, 1998.

LEWIS, B.A. et al. Computed tomographic evaluation of the canine intercondylar notch in normal and cruciate deficient stifles. Veterinary Comparative Orthopedics and Traumatology, v.21, p.119-124, 2008. Disponível em: <http:/ /dx.doi.org/10.3415/VCOT-07-04-0032http:// www.mendeley.com/research/computed-tomographicevaluation-canine-intercondylar-notch-normal-cruciatedeficient-stifles-1/>. Acesso em: 28 maio, 2010. doi: 10.3415/ VCOT-07-04-0032.

MANN, T.A. et al. The natural history of the intercondylar notch after notchplasty. American Journal of Sports Medicine, v.27, n.2, p.181-188, 1999.

MONTGOMERY, R.D. et al. Radiographic imaging of the canine intercondylar fossa. Veterinary Radiology and Ultrasound, v.36, n.4, p.276-282, 1995. Disponível em: <http://onlinelibrary.wiley.com/doi/10.1111/j.17408261.1995.tb00262.x/abstract>. Acesso em: 23 maio, 2010. doi: $10.1111 / \mathrm{j} .1740-8261.1995 . t b 00262 . x$

SELMI, A.L. et al. Clinical radiographic evaluation of a polyester prosthesis in dogs with cranial cruciate ligament rupture. Ciência Rural, v.32, n.5, p.793-798, 2002.

SELMI, A.L. et al. Incisuroplastia troclear e estabilização articular após transecção do ligamento cruzado cranial: estudo clínico e radiográfico em cães. Ciência Rural, v.37, n.5, p.1360-1366, 2007. Disponível em: <http://www.scielo.br/ scielo.php?script $=$ sci arttext \& pid = S 0103 84782007000500022>. Acesso em: 23 maio, 2010. doi: 10.1590/S0103-84782007000500022.

SOURYAL, T.O. et al. Bilaterality in anterior cruciate ligament injuries: associated intercondylar notch stenosis. American Journal of Sports Medicine, v.16, n.5, p.449-454, 1988.

SOURYAL, T.O.; FREEMAN, T.R. Intercondylar notch size and anterior cruciate injuries in athletes. A prospective study. American Journal of Sports Medicine, v.4, n.21, p.535539, 1993. Disponível em: <http://ajs.sagepub.com/content/ 16/5/449.full.pdf+html>. Acesso em: 01 jun. 2010. doi: $10.1177 / 036354658801600504$.

VASSEUR, P.B. et al. Evaluation of the Leeds-Keio synthetic replacement for the cranial cruciate ligament in dogs: an experimental study. Veterinary and Comparative Orthopedics and Traumatology, v.9, p.66-74, 1996. 